\title{
Poder preditivo do MoCa na avaliação neuropsicológica de pacientes com diagnóstico de demência
}

\author{
MoCa predictive power in neuropsychological assessment of patients with dementia
}



\section{Resumo}

O estudo teve como objetivo correlacionar testes neuropsicométricos em idosos com mais de quatro anos de escolaridade e avaliar a acurácia do MoCA no diagnóstico da doença de Alzheimer (DA) e comprometimento cognitivo leve (CCL). Foram avaliados 136 idosos atendidos no Instituto de Geriatria e Gerontologia, no período de abril de 2010 a dezembro de 2012. Os instrumentos utilizados foram o Miniexame do Estado Mental (MEEM), Cambridge Cognitive Examination (CAMCOG), teste do Desenho do Relógio (TDR), teste de Fluência Verbal, Escala de Depressão Geriátrica e Questionário de Atividades Funcionais de Pfeffer (QAFP), além do teste Montreal Cognitive Assessment (MoCA). Foi utilizada a análise de curva ROC para se estabelecer pontos de corte, e o coeficiente de correlação de Pearson, a fim de comparar o MoCA com os outros testes. Os resultados mostraram que o teste MoCA foi o melhor para diferenciar doença de Alzheimer dos casos de CCL. A sensibilidade e a especificidade encontradas foram, respectivamente, $82,2 \%$ e $92,3 \%$. A análise do teste de correlação evidenciou que o MoCA se correlacionou fortemente com outros testes já validados e de ampla aplicação no Brasil. Pode-se concluir que o MoCA mostrou ser o teste com maior valor preditivo para diferenciar DA de CCL e também diferenciar CCL dos controles normais. Além disso, o MoCA se correlacionou de maneira significativa com a variável idade e os testes MEEM, CAMCOG, TDR, de Fluência Verbal e QAFP, instrumentos já validados e amplamente utilizados no Brasil.

\section{Abstract}

This study aimed to correlate neuropsicometric tests in elderly over 4 years of schooling and assess MoCA accuracy in diagnosing Alzheimer's disease and mild cognitive impairment (MCI). It evaluated 136 elderly patients treated at the Institute of Geriatrics and Gerontology, from April 2010 to December 2012. The instruments used were the Mini-Mental State Examination (MMSE), Cambridge Cognitive Examination

\footnotetext{
Faculdade de Medicina de Jundiaí. Jundiaí, SP, Brasil.

2 Programa de Pós-graduação em Psicologia Educacional, Fundação Instituto de Ensino para Osasco. Centro Universitário FIEO. Osasco, SP, Brasil.
}

Palavras-chave: Avaliação Psicológica. Avaliação Neuropsicológica. Neuropsicologia. Psicodiagnóstico. Aprendizagem. 
(CAMCOG), Clock Drawing Test (CDT), Verbal Fluency test, Geriatric Depression Scale, Pfeffer Functional Activities Questionnaire (PFAQ), and Montreal Cognitive Assessement (MoCA). ROC curve analysis was used to establish cutoffs and the Pearson correlation coefficient to compare the MoCA with the other tests. The results showed that the MoCA was the best test to differentiate Alzheimer's disease from MCI. The sensitivity and specificity found were $82.2 \%$ and $92.3 \%$, respectively. The analysis of the correlation test showed that MoCA is strongly correlated with other tests already validated and wide applied in Brazil. The MoCA test showed the greatest predictive value to differentiate $\mathrm{AD}$ from MCI and also differ MCI from normal controls. Furthermore, MoCA was significantly correlated with the age variable and MMSE, CAMCOG, CDT, Verbal Fluency and PFAQ tests, instruments that are already validated and widely used in Brazil.
Key-words: Psychological

Assessment.

Neuropsychological

Assessment.

Neuropsychology.

Psychodiagnostic. Learning.

\section{INTRODUÇÃO}

A doença de Alzheimer (DA) acomete principalmente pessoas acima dos 60 anos de idade, não tem cura e o principal sintoma é a perda de memória recente. Sua evolução é progressiva e lenta, podendo durar até 20 anos, sendo classificada em: estágio leve, quando o paciente ainda apresenta áreas cognitivas preservadas, mantendo certa independência; e estágio avançado, quando a doença causa incapacidade completa e o paciente necessita de auxílio para alimentar-se, vestir-se ou tomar banho. ${ }^{1,2}$ A doença é onerosa para a família, pois dependendo da fase são necessárias duas pessoas para tomar conta do paciente, e para os sistemas de saúde, que arcam com custos de medicamentos e exames. ${ }^{3,4}$

Além do comprometimento de memória, o paciente pode apresentar, no início, afasia, apraxia ou problemas de coordenação visuo-motoraespacial. ${ }^{1}$ Com a evolução da doença, nos casos mais avançados, o comprometimento da região cortical torna o paciente incapaz de cuidar de si mesmo, por apresentar desorientação, confusão mental, além de provocar incontinência urinária.

Em outra perspectiva, declínios de memória definidos por testagem neuropsicológica, mas que não interferem com as atividades básicas e instrumentais da vida diária, dão origem a um novo construto, o denominado comprometimento cognitivo leve (CCL). Este é um importante alvo de recentes pesquisas, pois há a probabilidade de o quadro evoluir para demência - ou seja, o CCL é considerado uma fase transicional para a síndrome demencial, não sendo ainda assim caracterizado, mas indicando uma atenção mais detida neste sentido. ${ }^{5,6-9}$ Petersen et al. ${ }^{8}$ sugerem uma taxa de conversão de 10 a 15\% de CCL que evoluem para DA no primeiro ano de evolução. Essa taxa pode aumentar em $70 \%$ em até quatro anos após o diagnóstico da doença.

O CCL tem sido estudado desde a década de 1960 (com os relatos de Kral em 1962), quando foi descrito um quadro de declínio leve e intermitente de memória em pacientes idosos, denominando "esquecimento senil benigno". ${ }^{10}$ Em trabalho de revisão realizado por Dawe et al., ${ }^{11}$ observouse que nos últimos anos vários conceitos foram criados para tentar definir as condições clínicas e neuropsicológicas do comprometimento cognitivo leve, dentre os quais se podem citar: declínio cognitivo leve, transtorno cognitivo leve, alteração cognitiva leve, demência mínima, entre outros. Na primeira edição do Tratado de Geriatria e Gerontologia, foi destinada uma parte sobre as alterações biomédicas do envelhecimento, e o capítulo sobre o CCL foi intitulado Transtorno Cognitivo Leve (TCL), ${ }^{12}$ dado que corrobora a revisão de Dawe et al. ${ }^{11}$

O teste de Avaliação Cognitiva Montreal (Montreal Cognitive Assessement - MoCA) é um 
instrumento de triagem breve que avalia uma ampla gama de funções cognitivas (como as funções executivas, habilidades visuo-espaciais, nomeação, recuperação da memória, dígitos, sentença, raciocínio abstrato e orientação) necessários para contribuir com o diagnóstico do CCL e de demência. ${ }^{13}$ O tempo do teste é estimado em 20 minutos e a pontuação máxima possível é de 30 pontos. O ponto de corte para CCL é de 26 pontos e pontuação acima de 26 é considerada normal. ${ }^{13}$ Nasreddine et aI. ${ }^{13}$ evidenciaram, no estudo original do instrumento, $87 \%$ de especificidade do teste na sensibilidade para identificar idosos comprometidos e $90 \%$ em pessoas normais para detectar CCL. Além disso, a sensibilidade para detectar casos de DA com MoCA foi de $100 \% .{ }^{13}$ Devido a suas características psicométricas e utilidade nesses casos, este teste foi validado e adaptado em diferentes países. ${ }^{714-16}$ No Brasil, o estudo recente de Memória et al. ${ }^{17}$ indicou boas características psicométricas para esse teste.

Segundo os novos critérios de diagnóstico para a investigação da $\mathrm{DA}$, o uso de testes neuropsicológicos pode aumentar a compreensão sobre as capacidades preservadas desses idosos, ajudando a ajustar o tratamento. ${ }^{18,19}$ Sabe-se da existência de vários testes de triagem na área da Gerontologia e, geralmente, aqueles com maior precisão são usados. ${ }^{20}$ Nasreddine et aI. ${ }^{13}$ discutem os conceitos atuais de envelhecimento, especialmente CCL, e salientam que as novas ferramentas de avaliação se tornaram essenciais para essas pessoas, considerando CCL como um problema reconhecido com critérios diagnósticos específicos e que implica elevado risco de progressão para a DA.

Dentro desse contexto, o MoCA tem sido sugerido como instrumento eficaz para auxiliar no rastreamento do CCL e diagnóstico da DA. ${ }^{13}$ Sua simplicidade e administração rápida fazem com que seja útil em clínicas e hospitais, facilitando o trabalho da equipe de saúde, uma vez que abrange a maioria dos domínios cognitivos e tem alta sensibilidade para CCL. ${ }^{3}$ De acordo com Nasreddine et al., ${ }^{13}$ o MoCA pretende cumprir uma necessidade urgente que não tinha sido respondida por outros instrumentos de rastreamento disponíveis para detectar pacientes com CCL e diferenciá-los dos normais e idosos com DA.

Investigar sua utilidade na diferenciação da DA, CCL e idosos normais em uma amostra brasileira assume relevância, especialmente com idosos com mais de quatro anos de escolaridade, uma vez que esta é uma variável importante no diagnóstico. Vários estudos sugerem problemas nas funções cognitivas em diferenciar DA e CCL com níveis educacionais mais elevados, por causa do efeito teto de alguns testes cognitivos, ${ }^{21,22}$ aumentando a importância do presente estudo.

Assim, considerando a importância do diagnóstico de síndromes demenciais, bem como a importância do MoCA como teste de diagnóstico para demência e CCL, sendo este um instrumento amplamente utilizado no mundo, o objetivo deste estudo foi analisar a utilidade do MoCA na diferenciação do CCL, DA e idosos normais com mais de quatro anos de escolaridade.

\section{MÉTODOS}

A característica deste estudo é de corte transversal, com idosos que vivem na comunidade, com mais de quatro anos de escolaridade, de ambos os sexos e acima de 60 anos de idade. $\mathrm{O}$ protocolo do estudo foi previamente aprovado pelo Comitê de Ética em Pesquisa da Faculdade de Medicina de Jundiaí, e os sujeitos participaram da pesquisa por livre e espontânea vontade, através da assinatura do Termo de Consentimento Livre e Esclarecido, de acordo com o parecer de aprovação CEP no 54/11.

Adotaram-se os seguintes critérios de exclusão: pacientes com demência grave (Clinical Dementia Rating $\geq 3$ ); história de acidente vascular cerebral; paralisia em ambas as mãos; receber uma pontuação maior ou igual a 8 pontos na Escala de Depressão Geriátrica; parkinsonismo; tremor importante; e dificuldades visuais e auditivas. 
Participantes

Participaram deste estudo idosos atendidos em um Instituto de Geriatria e Gerontologia de um município do interior do Estado de São Paulo, ou seja, 5.668 idosos, sendo 1.853 atendidos no ano de 2010, 1.903 no ano de 2011 e 1.912 no ano de 2012. A seleção dos participantes ocorreu da seguinte maneira: do total acima descrito, apenas 508 foram encaminhados para a avaliação neuropsicométrica, de acordo com a anamnese realizada pelo geriatra do instituto. Dos 508 idosos submetidos a avaliação neuropsicológica, $57(11,22 \%)$ eram analfabetos e 306 (60,24\%) tinham escolaridade entre um e quatro anos, sendo, portanto, excluídos da pesquisa. Entre os outros 145 idosos, 62 (12,20\%) apresentavam escolaridade entre cinco e oito anos e 83 (16,34\%) acima de nove anos. Quando aplicados os critérios de exclusão, foram excluídos nove sujeitos, resultando, portanto, em 136 idosos incluídos no estudo. O fluxograma de seleção dos sujeitos é mostrado na figura 1.



Figura 1. Fluxograma de seleção de sujeitos para o estudo. Jundiaí-SP, 2010-2012.

Do total de 136 participantes, 52 idosos $(38,2 \%)$ receberam diagnóstico de DA, 45 $(33,1 \%)$ de CCL e $39(28,7 \%)$ fizeram parte do grupo controle (GC). Verificou-se que a grande maioria correspondeu ao sexo feminino, 89 $(65,4 \%)$, sendo a média de idade observada igual a 75,74 anos \pm 7,38 (mínimo=60; máximo=92). A amostra possuiu maior número de idosos com alta escolaridade, com 80 participantes (58,8\%) com mais de nove anos de estudo.

A média de idade do GC foi igual a 71,82 anos \pm 6,89 (mínimo=60; máximo=89), com a maioria correspondendo ao sexo feminino $(74,4 \%)$ e 27 idosos (69,2\%) apresentando mais de nove anos de estudo. Já o grupo com diagnóstico de DA apresentou média de idade igual a 77,92 anos \pm 6,97 (mínimo=64; máximo=91), com 63,5\% compostos por mulheres e 59,6\% apresentando alta escolaridade. Os participantes que receberam diagnóstico de CCL apresentavam média de idade igual a 76,60 anos \pm 7,06 (mínimo $=63$; máximo=92), 60\% correspondiam ao sexo feminino e 51,1\% apresentaram escolaridade entre cinco e oito anos. A frequência das variáveis "idade", "gênero" e "escolaridade" evidenciou 
grande participação do sexo feminino nos três grupos diagnósticos, alta escolaridade para o GC e DA e média de idade superior no grupo controle.

\section{Instrumentos e procedimentos}

Os pacientes foram submetidos a anamnese clínica detalhada, onde foram coletados exames laboratoriais e de neuroimagem, além de solicitada avaliação neuropsicométrica. Os testes neuropsicométricos aplicados foram: Montreal Cognitive Assessment (MoCA) versão brasileira, ${ }^{14}$ teste de Fluência Verbal (FV) de acordo com os critérios de Brucki et al., ${ }^{23}$ teste do Desenho do relógio ${ }^{24,25}$ e Miniexame do Estado Mental (MEEM). ${ }^{26}$ Foi realizada ainda a entrevista estruturada do Cambrigde Examinations for Mental Disorders of the Elderly (CAMDEX) e sua bateria cognitiva Cambrigde Cognitive Examination (CAMCOG) ${ }^{27}$ Para avaliar os sintomas depressivos, foi aplicada a Escala de Depressão Geriátrica (EDG) abreviada, com 15 itens. ${ }^{28} \mathrm{E}$ para a análise do desempenho de vida diária, foi utilizado o Questionário de Atividades Funcionais de Pfeffer (PFAQ). ${ }^{23}$

Os critérios diagnósticos para demência foram baseados no DSM-IV, ${ }^{24}$ sendo que NINCDSADRDA (National Institute of Neurological and Communicative Disorders and Stroke - Alsheimer's Disease and Related Disorders Association) ${ }^{19}$ foi utilizado como critério para DA. Para o CCL, o diagnóstico foi clínico, mas também foram utilizados os critérios de Petersen et al. ${ }^{8}$ para auxiliar o diagnóstico. Foram incluídos nesta pesquisa apenas pacientes com CCL amnéstico, ou seja, idosos que só apresentavam comprometimento da memória. Além disso, não foi feito o cálculo de 1,5 desviopadrão, como sugerido por Petersen et al., ${ }^{8}$ sendo analisados para o diagnóstico os subitens de memória do MEEM e do CAMCOG. Os itens de memória do MEEM considerados são os das três palavras ("vaso", "carro" e "janela"); e do CAMCOG são os das seis figuras ("sandália", "máquina de escrever", "balança", "mala", "relógio" e "abajur").
Os critérios de inclusão para os idosos do grupo controle (GC) foram: pontuar acima do ponto de corte nos testes neuropsicológicos (pontuar acima de 80 pontos no CAMCOG); não ter sintomas de demência (não apresentar dificuldade em atividades de vida diária); pontuação da EDG inferior a 7; e não ter comprometimento em desempenhar atividades de vida diária de acordo com o Questionário de Atividades Funcionais de Pfeffer (QAFP). ${ }^{29,30}$ Após a avaliação clínica e neuropsicológica que o paciente realizou, foi entrevistado um informante ou um familiar com convívio diário com o idoso, a fim de relatar possíveis sintomas, acrescentar outras informações relevantes ou relatar mudanças de comportamento.

Foram realizadas análises descritivas quanto a idade, gênero e escolaridade. As variáveis foram descritas por meio de frequências e médias com desvio-padrão. Por meio do teste KolmogorovSmirnov, verificou-se a distribuição (paramétrica ou não paramétrica) da amostra em todas as medidas efetuadas. Também foram estabelecidos, por meio de análises com a curva ROC, pontos de corte para os escores do MoCA, CAMCOG, MEEM, TDR e FV. Em relação às análises comparativas entre os testes neuropsicométricos, foram utilizados coeficientes de correlação de Pearson $(r)$.

\section{RESULTADOS}

As análises da curva ROC evidenciam que o MoCA foi o teste com maior valor de área sob a curva (AUC), sugerindo que este é o teste mais eficaz para diferenciar os grupos CCL e GC. A sensibilidade e especificidade encontradas, respectivamente, foram de $82,2 \%$ e $92,3 \%$ (tabelas 1 e 2). Verifica-se que o MoCA foi diferente do TDR escala de Mendez \& Shulman, sendo a sensibilidade estatisticamente diferente.

O MoCA também se mostrou importante em diferenciar o grupo de controles normais e os de DA, com 98,1\% de sensibilidade e 100\% de especificidade. Observa-se que o MoCA foi 
diferente de todos os demais testes, apresentando sensibilidade estatisticamente diferente dos citados (sendo do MoCA a maior) e maior especificidade, ou seja, o MoCA é superior aos demais, em diferenciar DA de controles normais.

De acordo com os pontos de corte apresentados no MoCA, para diferenciação dos grupos diagnósticos, pela metodologia da curva ROC, encontra-se um escore abaixo de 21 pontos para DA, e para CCL entre 21 e 24 pontos. $O$ ponto de corte que diferencia GC do CCL é a pontuação obtida, que foi maior, 25 pontos.

As análises com o teste de correlação de Pearson evidenciam que o teste MoCA possui correlação de forte a moderada, positiva e significativa com o MEEM $(r=0,81 ; \mathrm{p}<0,0001)$, CAMCOG $(r=0,86 ; \mathrm{p}<0,0001)$ e fluência verbal versão frutas $(r=0,70 ; \mathrm{p}<0,0001)$. Os resultados também apontam correlação moderada, positiva e significativa entre o MoCA e o TDR, escala de
Mendez $(r=0,51 ; \mathrm{p}<0,0001)$ e escala de Shulman $(r=0,59 ; \mathrm{p}<0,0001), \mathrm{FV}$ versão animais $(r=0,58$; $\mathrm{p}<0,0001)$ e versão palavras com a letra " $\mathrm{M}$ " $(r=0,57 ; \mathrm{p}<0,0001)$. Houve correlação moderada, negativa e significativa entre o MoCA e o QAFP $(r=-0,67 ; \mathrm{p}<0,0001)$, sugerindo que quanto menor a pontuação do MoCA, maior será o escore do QAFP - ou seja, maior será a dependência em desempenhar as atividades de vida diária quando o paciente apresentar maior comprometimento cognitivo.

Em relação a escolaridade e idade, pode-se observar, na tabela 2, que não houve correlação significativa entre o MoCA e a variável "escolaridade" $(r=0,14 ; \mathrm{p}=0,111)$. Encontra-se correlação moderada, positiva e significativa entre o MoCA e a variável "idade" $(r=0,40$; $\mathrm{p}=0,0001)$, o que evidencia que quanto maior a idade do paciente, menor será a pontuação encontrada no teste MoCA. 
Tabela 1. Pontos de corte, sensibilidade e especificidade calculados pela metodologia de curva ROC para diferenciar CCL e controles (C). Jundiaí-SP, 2010-2012.

\begin{tabular}{|c|c|c|c|c|c|c|c|c|}
\hline \multirow{3}{*}{ MoCA } & \multirow{2}{*}{$\begin{array}{c}\mathrm{CCL} \\
\mathrm{n}\end{array}$} & \multirow{2}{*}{$\begin{array}{l}\mathrm{C} \\
\mathrm{n}\end{array}$} & \multirow{2}{*}{\multicolumn{2}{|c|}{$\begin{array}{c}\text { Sensibilidade } \\
\text { IC } 95 \%\end{array}$}} & \multicolumn{2}{|c|}{ Especificidade } & \multirow[b]{2}{*}{ ASC } & \multirow[b]{2}{*}{$\mathrm{p}$} \\
\hline & & & & & \multicolumn{2}{|c|}{ IC $95 \%$} & & \\
\hline & & & & & & & & $<0,0001$ \\
\hline$<25$ & 37 & 3 & \multicolumn{2}{|c|}{$82,2 \%$} & \multicolumn{2}{|c|}{$92,3 \%$} & 0,943 & \\
\hline$>=25$ & 8 & 36 & $71,1 \%$ & $93,4 \%$ & $83,9 \%$ & $100,0 \%$ & & \\
\hline CAMCOG & & & & & & & & $<0,0001$ \\
\hline$<92$ & 30 & 4 & \multicolumn{2}{|c|}{$66,7 \%$} & \multicolumn{2}{|c|}{$89,7 \%$} & 0,8621 & \\
\hline$>=92$ & 15 & 35 & $52,9 \%$ & $80,4 \%$ & $80,2 \%$ & $99,3 \%$ & & \\
\hline GDS & & & & & & & & 0,2044 \\
\hline$>=2$ & 27 & 18 & \multicolumn{2}{|c|}{$60,0 \%$} & \multicolumn{2}{|c|}{$53,8 \%$} & 0,5729 & \\
\hline$<2$ & 18 & 21 & $45,7 \%$ & $74,3 \%$ & $38,2 \%$ & $69,5 \%$ & & \\
\hline MEEM & & & & & & & & $<0,0001$ \\
\hline$<29$ & 36 & 7 & \multicolumn{2}{|c|}{$80,0 \%$} & \multicolumn{2}{|c|}{$82,1 \%$} & 0,8322 & \\
\hline$>=29$ & 9 & 32 & $68,3 \%$ & $91,7 \%$ & $70,0 \%$ & $94,1 \%$ & & \\
\hline Mendez & & & & & & & & 0,0049 \\
\hline$<20$ & 30 & 14 & \multicolumn{2}{|c|}{$66,7 \%$} & \multicolumn{2}{|c|}{$64,1 \%$} & 0,6781 & \\
\hline$>=20$ & 15 & 25 & $52,9 \%$ & $80,4 \%$ & $49,0 \%$ & $79,2 \%$ & & \\
\hline PFAQ & & & & & & & & $<0,0001$ \\
\hline$>=1$ & 34 & 9 & \multicolumn{2}{|c|}{$75,6 \%$} & \multicolumn{2}{|c|}{$76,9 \%$} & 0,7752 & \\
\hline$<1$ & 11 & 30 & $63,0 \%$ & $88,1 \%$ & $63,7 \%$ & $90,1 \%$ & & \\
\hline Shulman & & & & & & & & 0,0045 \\
\hline$<5$ & 29 & 13 & \multicolumn{2}{|c|}{$64,4 \%$} & \multicolumn{2}{|c|}{$66,7 \%$} & 0,6658 & \\
\hline$>=5$ & 16 & 26 & $50,5 \%$ & $78,4 \%$ & $51,9 \%$ & $81,5 \%$ & & \\
\hline
\end{tabular}

Legenda: Comprometimento Cognitivo Leve (CCL); ASC - área sobre a curva (estimativa de intervalo da variação); Montreal Cognitive Assessment (MoCA); Cambrigde Cognitive Examination (CAMCOG); Escala de Depressão Geriátrica (GDS); Miniexame do Estado Mental (MEEM); Teste do Desenho do Relógio (critério - Mendez); Questionário de Atividades Funcionais de Pfeffer (QAFP); Teste do Desenho do Relógio (critério - Shulman). 
Tabela 2. Pontos de corte, sensibilidade e especificidade calculados pela metodologia de curva ROC para diferenciar DA e controles (C). Jundiaí-SP, 2010-2012.

\begin{tabular}{|c|c|c|c|c|c|c|c|c|}
\hline \multirow{2}{*}{\multicolumn{2}{|c|}{$\begin{array}{c}\mathrm{DA} \\
\mathrm{n}\end{array}$}} & $\mathrm{C}$ & \multirow{2}{*}{\multicolumn{2}{|c|}{$\begin{array}{l}\text { Sensibilidade } \\
\text { IC } 95 \%\end{array}$}} & \multicolumn{2}{|c|}{ Especificidade } & \multirow[b]{2}{*}{ ASC } & \multirow[b]{2}{*}{$\mathrm{p}$} \\
\hline & & $\mathrm{n}$ & & & & & & \\
\hline $\mathrm{MoCA}$ & & & & & & & & $<0,0001$ \\
\hline$<23$ & 51 & 0 & \multicolumn{2}{|c|}{$98,1 \%$} & \multicolumn{2}{|c|}{$100,0 \%$} & 0,9993 & \\
\hline$>=23$ & 1 & 39 & $94,3 \%$ & $101,8 \%$ & $100,0 \%$ & $100,0 \%$ & & \\
\hline CAMCOG & & & & & & & & $<0,0001$ \\
\hline$<90$ & 47 & 1 & \multicolumn{2}{|c|}{$90,4 \%$} & \multicolumn{2}{|c|}{$97,4 \%$} & 0,9712 & \\
\hline$>=90$ & 5 & 38 & $82,4 \%$ & $98,4 \%$ & $92,5 \%$ & $102,4 \%$ & & \\
\hline GDS & & & & & & & & 0,4232 \\
\hline$>=3$ & 23 & 14 & \multicolumn{2}{|c|}{$44,2 \%$} & \multicolumn{2}{|c|}{$64,1 \%$} & 0,5754 & \\
\hline$<3$ & 29 & 25 & $30,7 \%$ & $57,7 \%$ & $49,0 \%$ & $79,2 \%$ & & \\
\hline MEEM & & & & & & & & $<0,0001$ \\
\hline$<29$ & 48 & 7 & \multicolumn{2}{|c|}{$92,3 \%$} & \multicolumn{2}{|c|}{$82,1 \%$} & 0,9522 & \\
\hline$>=29$ & 4 & 32 & $85,1 \%$ & $99,6 \%$ & $70,0 \%$ & $94,1 \%$ & & \\
\hline Mendez & & & & & & & & $<0,0001$ \\
\hline$<19$ & 33 & 4 & \multicolumn{2}{|c|}{$63,5 \%$} & \multicolumn{2}{|c|}{$89,7 \%$} & 0,8119 & \\
\hline$>=19$ & 19 & 35 & $50,4 \%$ & $76,5 \%$ & $80,2 \%$ & $99,3 \%$ & & \\
\hline PFAQ & & & & & & & & $<0,0001$ \\
\hline$>=2$ & 46 & 6 & \multicolumn{2}{|c|}{$88,5 \%$} & \multicolumn{2}{|c|}{$84,6 \%$} & 0,9453 & \\
\hline$<2$ & 6 & 33 & $79,8 \%$ & $97,1 \%$ & $73,3 \%$ & $95,9 \%$ & & \\
\hline Shulman & & & & & & & & $<0,0001$ \\
\hline$<5$ & 43 & 13 & \multicolumn{2}{|c|}{$82,7 \%$} & \multicolumn{2}{|c|}{$66,7 \%$} & 0,8141 & \\
\hline$>=5$ & 9 & 26 & $72,4 \%$ & $93,0 \%$ & $51,9 \%$ & $81,5 \%$ & & \\
\hline
\end{tabular}

Legenda: Comprometimento Cognitivo Leve (CCL); ASC - área sobre a curva (estimativa de intervalo da variação); Montreal Cognitive Assessment (MoCA); Cambrigde Cognitive Examination (CAMCOG); Escala de Depressão Geriátrica (GDS); Miniexame do Estado Mental (MEEM); Teste do Desenho do Relógio (critério - Mendez); Questionário de Atividades Funcionais de Pfeffer (QAFP); Teste do Desenho do Relógio (critério - Shulman).

\section{DISCUSSÃO}

Os objetivos deste estudo foram correlacionar testes neuropsicométricos em idosos com mais de quatro anos de escolaridade com diagnóstico de doença de Alzheimer e comprometimento cognitivo leve; e contribuir com o diagnóstico diferencial, analisando a eficácia do MoCA em detrimento dos demais testes em amostra de pacientes idosos atendidos no Instituto de Geriatria e Gerontologia. É evidente o papel dos anos de estudo no desempenho em testes cognitivos, ${ }^{21,23,31,32}$ que, além de proporcionar reserva cognitiva, funciona como mecanismo protetor de quadros demenciais. ${ }^{33,34}$ De acordo com Parente et al. ${ }^{33}$ e Nitrini et al. ${ }^{34}$ o fato de a escolaridade exercer influência sobre a cognição corrobora os resultados desta pesquisa, em que 
a maior porcentagem de alta escolaridade (acima de nove anos) foi encontrada no GC, ou seja, em indivíduos sem comprometimento cognitivo.

Em relação ao desempenho cognitivo dos idosos, muitos estudos apontam para a importância das avaliações neuropsicométricas no diagnóstico diferencial das síndromes demenciais, principalmente no que se refere a aspectos da aprendizagem e da memória. ${ }^{35-37}$ Gainotti et al. ${ }^{35}$ encontraram diferenças significativas entre DA e pseudodemência depressiva em relação ao desempenho cognitivo em tarefas que avaliam a memória imediata e erros de intrusão na memória tardia. Em outras demências, Giovannetti et al. ${ }^{36}$ compararam a demência vascular com a DA e verificaram que, no caso das demências vasculares, há o comprometimento na formação de conceitos e, no caso da DA, esses déficits são secundários ao comprometimento da resposta verbal. Com relação a este comprometimento, Gold et al. ${ }^{37}$ sugerem que pacientes com DA apresentam disfunção do hipocampo e também do córtex pré-frontal, por apresentarem declínio da fluência.

Os estudos acima descritos apontam uma preocupação comum, que é a busca de um teste com maior valor preditivo para o diagnóstico diferencial dos comprometimentos cognitivos. ${ }^{35-38}$ Considerando o teste MoCA, os dados desta pesquisa evidenciam que a maioria de seus itens consegue diferenciar os três grupos diagnósticos. Vale ressaltar que o MoCA foi desenvolvido como ferramenta de avaliação diagnóstica para o CCL e casos leves de demência. ${ }^{13}$ Quando comparado ao MEEM (um dos instrumentos de rastreio mais utilizados em todo o mundo), o MoCA possui mais itens de memória em sua avaliação, além de aspectos de função executiva ${ }^{13}$ e com um subitem de linguagem mais estruturado. ${ }^{39}$

Nesta pesquisa, ficou evidenciada a melhor acurácia diagnóstica do MoCA, quando comparado a MEEM, CAMCOG, FV e TDR. Outros estudos também comprovam a eficácia do MoCA como ferramenta de auxílio para o diagnóstico diferencial. Dong et al. ${ }^{40}$ analisaram 230 idosos com CCL e seus resultados mostraram que o MoCA apresentou uma área sobre a curva (AUC) significativamente superior quando comparado ao MEEM - respectivamente, 0,92 e 0,84 . Os dados corroboram os achados desta pesquisa, em que a detecção dos três grupos diagnósticos foi mais bem avaliada pelo MoCA do que pelos outros instrumentos.

Outro estudo que reforça a hipótese de o MoCA ser uma ferramenta eficaz para o diagnóstico de declínio cognitivo foi realizada por Markwick et al., ${ }^{41}$ que observaram que mesmo em pacientes com comprometimento cognitivo, os escores do MEEM apresentavam-se normais, enquanto que os escores do MoCA apontavam declínio. Os resultados apontavam declínio evidente nos subtestes do MoCA, e a conclusão a que os pesquisadores chegaram foi que o MoCA parece ser um teste de rastreio mais sensível para detectar declínio cognitivo precocemente.

Esse dado está de acordo com o encontrado nesta pesquisa, na qual o MoCA foi o teste com maior sensibilidade e especificidade em diferenciar o CCL dos casos de DA e de idosos normais. A escolaridade também reforça a hipótese de um melhor desempenho no MoCA, quando comparado aos outros instrumentos, pois a amostra corresponde a idosos acima de quatro anos de estudo.

Sobre a melhor eficácia do MoCA, outra hipótese consideraria o próprio teste. Como descrito anteriormente, o MoCA é um teste mais estruturado que o MEEM, com mais itens de memória, aspectos relevantes de linguagem e conta com avaliação das funções executivas. ${ }^{13,39}$ Siedlecki et al. ${ }^{42}$ argumentam que a reserva cognitiva está fortemente correlacionada com as funções executivas. Por ser um teste de rastreio que abrange aspectos fundamentais da cognição e por permitir avaliação das funções executivas, o MoCA apresentou-se como instrumento eficaz para idoso com alta escolaridade e declínio cognitivo leve. Os resultados com o coeficiente 
de correlação de Pearson apontaram forte correlação entre o MoCA e outros instrumentos já validados e amplamente utilizados no Brasil.

Entre o MEEM e o CAMCOG, a correlação com o MoCA foi igual a 0,81 e 0,86 , respectivamente. Os dados desta pesquisa mostram correlação mais robusta quando comparada ao trabalho feito por Memória et al., ${ }^{17}$ que encontraram correlação do MoCA entre o CAMCOG e MEEM igual a 0,82 e 0,74 , respectivamente. A correlação robusta e significativa entre MEEM, TDR, CAMCOG e FV sugere que o MoCA parece ser um teste adequado para ser aplicado em idosos com mais de quatro anos de estudo.

De fato, a existência de forte interrelação entre os escores dos testes sugere a possibilidade de estarem aferindo aspectos cognitivos semelhantes. Todavia, o fato de um teste ser melhor que o outro na discriminação dos quadros clínicos pode indicar que, apesar de aferirem aspectos semelhantes, o fazem em níveis de dificuldade distintos. Isso é esperado, já que a natureza das tarefas e a forma de avaliações são também diferentes entre os instrumentos, revelando que algumas provas (as do MoCA sobretudo) tendem a ser mais adequadas na discriminação de CCL e DA quanto aos aspectos cognitivos aferidos, que são equivalentes aos aferidos no MEEM e CAMCOG.

Outro dado importante e evidente no teste de correlação foi a afinidade significativa entre o MoCA e o QAFP. A relação entre comprometimento das atividades de vida diária e a demência está bem descrita na literatura tanto nacional quanto internacional. ${ }^{18,19,33,34}$ É importante destacar que o desempenho nas atividades de vida diária é um aspecto também exigido pelos critérios diagnósticos, o que direciona os profissionais da área da saúde em combinar as escalas funcionais com os teste cognitivos. $^{18,19}$ Abreu et al. $^{43}$ referem que a memória é um item fundamental em relação aos processos cognitivos e se correlaciona diretamente com as atividades do cotidiano.
Seguindo os resultados deste estudo, bem como as discussões que foram tecidas em relação aos mesmos, estes demonstraram ser necessário correlacionar um teste cognitivo e uma escala de atividades funcionais na investigação das síndromes demenciais. Segundo Bustamante et al., ${ }^{44}$ a combinação entre testes cognitivos e questionários que avaliam a funcionalidade aumenta a precisão diagnóstica nos casos de demência. Abreu et $a_{1}{ }^{43}$ evidenciam que $\mathrm{O}$ teste MEEM e a bateria cognitiva CAMCOG, consideradas as avaliações mais utilizadas em nosso meio, parecem se correlacionar com as escalas de funcionalidade IQCODE e o índice Katz.

Outro estudo que corrobora os dados encontrados nesta pesquisa foi realizado por Marra et al. $^{45}$ em amostra de Minas Gerais, utilizando as escalas Lawton, o índice Katz e o Questionário de Atividades Funcionais de Pfeffer. A pesquisa evidenciou que os idosos apresentavam maior dificuldade em desempenhar atividades de vida diária quanto maior o nível de demência. Os resultados da correlação desta pesquisa estão em concordância com a literatura encontrada. ${ }^{18,19,33,34,43-45}$

Estudos com amostras maiores devem ser feitos, pois esta foi uma limitação deste estudo. Também estudos com níveis de escolaridade mais baixos podem ser feitos para analisar as relações ora estabelecidas.

\section{CONCLUSÕES}

Pode-se concluir que o Montreal Cognitive Assessment (MoCA) demonstrou ser um instrumento com maior valor preditivo para diferenciar doença de Alzheimer (DA) de comprometimento cognitivo leve (CCL), e também diferenciar CCL dos controles normais. Além disso, o MoCA se correlacionou de maneira significativa com a variável "idade" e os testes Miniexame do Estado Mental (MEEM), Cambrigde Cognitive Examination(CAMCOG), Teste do Desenho do Relógio (TDR), Fluência Verbal 
(FV) e Questionário de Atividades Funcionais de Pfeffer (QAFP), que são instrumentos já validados e amplamente utilizados no Brasil.

Sugerem-se novos estudos, de modo a aferir tais achados, bem como com populações de outras localidades, tais como de outras regiões do país, uma vez que é sabido que fatores regionais propiciam habilidades e/ou dificuldades específicas para essa população. ${ }^{44,45}$ Com isso, identifica-se a relevância do uso de testes funcionais como auxiliares no diagnóstico de demência e até mesmo para avaliar outros fatores incapacitantes nesses quadros.

\section{REFERÊNCIAS}

1. Damasceno BP. Demências. In: Guariento MH, Neri AL, organizadores. Assistência ambulatorial ao Idoso. Campinas: Alínea; 2010. p. 243-54.

2. Silva DW, Damasceno BP. Demência na população de pacientes do Hospital das Clínicas da Unicamp. Arq Neuropsiquiatr 2002;60(4):996-9.

3. Hamdan AC. Avaliação neuropsicológica na doença de Alzheimer e no comprometimento cognitivo leve. Psicol Argum 2008;26(54):183-92.

4. Veras RP, Caldas CP, Dantas SB, Sancho LG, Sicsu B, Motta LB, et al. Avaliação dos gastos com o cuidado do idoso com demência. Rev Psiquiatr Clín 2007;34(1):5-12.

5. Teixeira AL, Caramelli P. Neuropsicologia das demências. In: Fuentes D, Malloy-Diniz LF, Camargo CP, Cosenza RM, organizadores. Neuropsicologia: teoria e prática. Porto Alegre: Artmed; 2008. p. 356-63.

6. Morris JC, Storandt M, Miller JP, McKeel DW, Price JL, Rubin EH, et al. Mild cognitive impairment represents early-stage Alzheimer disease. Arch Neurol 2001;58(3):397-405.

7. Rahman TT, El Gaafary MM. Montreal Cognitive Assessment Arabic version: reliability and validity prevalence of mild cognitive impairment among elderly attending geriatric clubs in Cairo. Geriatr Gerontol Int 2009;9(1):54-61.

8. Petersen RC, Stevens J, Ganguli M, Tangalos EG, Cummings J, DeKosky ST. Practice parameter: early detection of dementia: mild cognitive impairment (an evidence-based review). Neurology 2001;56:1133-42.

9. Taragano FE, Allegri RF, Lyketsos C. Mild behavioral impairment: a prodromal stage of dementia. Dement Neuropsychol 2008;2(4):256-60.

10. Bottino CMC, Moreno MDPQ. Comprometimento cognitivo leve: critérios diagnósticos e validade clínica. In: Bottino CMC, Laks J, Blay SL, organizadores. Demência e transtornos cognitivos em idosos. Rio de Janeiro: Guanabara Koogan; 2006. p. 31-7.
11. Dawe B, Procter A, Philpot M. Concepts of mild memory impairment in the elderly and their relationship to dementia: a review. Int J Geriatr Psychiatry 1992;7(7):473-9.

12. Canineu PR, Bastos A. Transtorno cognitivo leve. In: Freitas EV, Py L, Neri AL, Cançado FAX, Gorzoni ML, Rocha SM, organizadores. Tratado de Geriatria e Gerontologia. Rio de Janeiro: Guanabara Koogan; 2002. p. 128-32.

13. Nasreddine ZS, Phillips NA, Bédirian V, Charbonneau S, Whitehead V, Collin I, et al. The Montreal Cognitive Assessment, MoCA: a brief screening tool for mild cognitive impairment. J Am Geriatr Soc 2005;53(4):695-9.

14. Sarmento ARL. Apresentação e aplicabilidade da versão brasileira da MoCA (Montreal Cognitive Assessment) para rastreio de comprometimento cognitivo leve [dissertação]. São Paulo: Escola Paulista de Medicina da Universidade Federal de São Paulo. Programa de Pós-graduação; 2009.

15. Tsai CF, Lee WJ, Wang SJ, Shia BC, Nasreddine Z, Fuh JL. Psychometrics of the Montreal Cognitive Assessment (MoCA) and its subscales: validation of the Taiwanese version of the MoCA and an item response theory analysis. Int Psychogeriatr 2012;24(4)12:651-8.

16. Freitas S, Simões MR, Alves L, Santana I. Montreal Cognitive Assessment: validation study for Mild Cognitive Impairment and Alzheimer Disease. Alzheimer Dis Assoc Disord 2013;27(1):37-43.

17. Memória CM, Yassuda MS, Nakano, EY, Forlenza OV. Brief screening for mild cognitive impairmente: validation of brazilian version of the Montreal Cognitive Assessment. Int J Geriatr Psychiatry. [Epub ahead of print] 2012.

18. Albert MS, DeKosky ST, Dickson D, Dubois B, Feldman HH, Fox NC, et al. The diagnosis of mild cognitive impairment due to Alzheimer's disease: recommendations from the National Institute 
on Aging-Alzheimer's Association workgroups on diagnostic guidelines for Alzheimer's disease. Alzheimers Dement 2011;7(3):270-9.

19. McKhann GM, Knopman DS, Chertkow H, Hyman BT, Jack CR Jr, Kawas CH, et al. The diagnosis of dementia due to Alzheimer's disease: recommendations from the National Institute on Aging-Alzheimer's Association workgroups on diagnostic guidelines for Alzheimer's disease. Alzheimers Dement 2011;7(3):263-9.

20. Heinik J, Solomesh I, Berkman P. Correlation between the CAMCOG, the MMSE, and three clock drawing tests in a specialized outpatient psychogeriatric service. Arch Gerontol Geriatr 2004;38(1):77-84.

21. Cecato JF, Fiorese B, Montiel JM, Bartholomeu D, Martinelli JE. Clock drawing test in elderly individuals with different education levels: correlation with clinical dementia rating. Am J Alzheimers Dis Other Demen 2012;27(8):620-4.

22. Cecato JF, Martinelli JO, Montiel JM, Bartholomeu $\mathrm{D}$, Aramaki FO. Discriminative power of Montreal Cognitive Assessment. JMED Res 2014;1:1-10.

23. Brucki SMD, Nitrini R, Caramelli P, Bertolucci PHF, Okamoto IH. Sugestões para o uso do Mini-Exame do Estado Mental no Brasil. Arq Neuropsiquiatr 2003;61(3 B):777-81.

24. Mendez MF, Ala T, Underwood K. Development of scoring criteria for the clock drawing task in Alzheimer's disease. J Am Geriatr Soc 1992;40(11):1095-99.

25. Shulman KI, Gold DP, Cohen CA, Zucchero CA. Clock-drawing and dementia in the community: a longitudinal study. Int J Geriatr Psychiatry 1993;8(6):487-96.

26. Folstein MF, Folstein SE, McHugh PR. Mini-Mental State: a practical method for grading the cognitive state of patients for the clinician. J Psychiatr Res 1975;12(3):189-18.

27. Roth M, Tym E, Mountjoy CQ, Huppert FA, Hendrie H, Verma S, Goddard R. CAMDEX. A standardised instrument for the diagnosis of mental disorder in the elderly with special reference to the early detection of dementia. Br J Psychiatr 1986;149:698-709.

28. Yesavage JA, Brink TL, Rose TL, Lum O, Huang $\mathrm{V}$, Adey M, et al. Development and validation of a geriatric depression screening scale: a preliminary report. J Psychiatr Res 1983;17(1):37-49.
29. American Psychiatry Association. Diagnostic and statistical manual of mental disorders. $4^{\text {th }}$ ed. Washington, DC: American Psychiatry Association; 1994.

30. Pfeffer RI, Kurosaki TT, Harrah CH Jr, Chance JM. Measurement of functional activities in older adults in the community. J Gerontol 1982;37(3):323-9.

31. Diniz BSO, Volpe FM, Tavares AR. Nível educacional e idade no desempenho do Mini exame do Estado Mental em idosos residentes na comunidade. Rev Psiquiatr Clín 2007;34(1):13-17.

32. Moraes C, Pinto JA Jr, Lopes MA, Litvoc J, Bottino CM. Impact of sociodemographic and health variables on mini-mental state examination in a communitybased sample o folder people. Eur Arch Psychiatr Clin Neurosci 2010;260(7):535-42.

33. Parente MAMP, Scherer LC, Zimmermann N, Fonseca RP. Evidências do papel da escolaridade na organização cerebral. Rev Neuropsicol Latinoam 2009;1(1):72-80.

34. Nitrini R, Bottino CMC, Albala C, Capunay NSC, Ketzoian C, Rodriguez JJL, et al. Prevalence of dementia in Latin America: a collaborative study of population-based cohorts. Int Psychogeriatr 2009;21(4):622-30.

35. Gainotti G, Marra C. Some aspects of memory disorders clearly distinguish dementia of the Alzheimer's type from depressive pseudo-dementia. J Clin Exp Neuropsychol 1994;16(1):65-78.

36. Giovannetti T, Lamar M, Cloud BS, Swenson R, Fein D, Kaplan E, et al. Different underlying mechanisms for deficits in concept formation in dementia. Arch Clin Neuropsychol 2001;16(6):547-60.

37. Gold CA, Marchant NL, Koutstaal W, Schacter DL, Budson AE. Conceptual fluency at test shifts recognition response bias in Alzheimer's disease: implications for increased false recognition. Neuropsychologia 2007;45(12):2791-801.

38. Valls-Pedret C, Olives J, Bosch B, Caprile C, Castellví M, Molinuevo JL, et al. Landscape test for assessing visual memory in Alzheimer's disease. Rev Neurol 2011;53(1):1-7.

39. Cecato JF, Martinelli JE, Bartholomeu LL, Basqueira AP, Yassuda MS, Aprahamian I. Verbal behavior in Alzheimer's disease patients: analysis in repetition phrases. Dement Neuropsychol 2010;4(3):202-6.

40. Dong Y, Lee WY, Basri NA, Collinson SL, Merchant RA, Venketasubramanian N, et al. The Montreal Cognitive Assessment is superior to the Mini-mental 
State Examination in detecting patients at higher risk of dementia. Int Psychogeriatr 2012;24(11):1749-55.

41. Markwick A, Zamboni G, De Jager CA. Profiles of cognitive subtest impairment in the Montreal Cognitive Assessment (MoCA) in a research cohort with normal Mini-mental State Examination (MMSE) scores. J Clin Exp Neuropsychol 2012;34(7):750-7.

42. Siedlecki KL, Stem Y, Reuben A, Sacco RL, Elkind MSV, Wright CB. Construct validity of cognitive reserve in a multiethnic cohort: the Northern Manhattan Study. J Int Neuropsychol Soc 2009;15(4):558-69.
43. Abreu ID, Forlenza OV, Barros HL. Demência de Alzheimer: correlação entre memória e autonomia. Rev Psiquiatr Clín 2005;32(3):131-6.

44. Bustamante SEZ, Bottino CMC, Lopes MA, Azevedo D, Hototian SR, Litvoc J, et al. Instrumentos combinados na avaliação de demência em idosos: resultados preliminares. Arq Neuropsiquiatr 2003;61(3A):601-6.

45. Marra TA, Pereira LSM, Faria CDCM, Pereira DS, Martins MAA, Tirado MGA. Avaliação das atividades de vida diária de idosos com diferentes níveis de demência. Rev Bras Fisioter 2007;11(4):267-73. 